\title{
Absence of angiotensin II type 1 receptor in bone marrow-derived cells is detrimental in the evolution of renal fibrosis
} \author{
Agnes B. Fogo, ${ }^{1,3,4}$ Jeffrey M. Davidson, ${ }^{3}$ MacRae F. Linton, ${ }^{4}$ Sergio Fazio, ${ }^{4}$ \\ Toshio Homma, ${ }^{2}$ Hiroaki Yoshida, ${ }^{1,4}$ and Iekuni Ichikawa ${ }^{1,2,4}$ \\ ${ }^{1}$ Department of Pediatrics, Vanderbilt University Medical Center, Nashville, Tennessee, USA \\ ${ }^{2}$ Department of Pediatrics, Tokai University School of Medicine, Isehara, Japan \\ ${ }^{3}$ Department of Pathology, and \\ ${ }^{4}$ Department of Medicine, Vanderbilt University Medical Center, Nashville, Tennessee, USA
}

Masashi Nishida, ${ }^{1}$ Hidehiko Fujinaka, ${ }^{1}$ Taiji Matsusaka, ${ }^{1,2}$ James Price, ${ }^{3}$ Valentina Kon, ${ }^{1}$

\begin{abstract}
We examined the in vivo function of the angiotensin II type 1 receptor (Agtr1) on macrophages in renal fibrosis. Fourteen days after the induction of unilateral ureteral obstruction (UUO), wild-type mice reconstituted with marrow lacking the Agtr1 gene (Agtr $\left.1^{-/-}\right)$developed more severe interstitial fibrosis with fewer interstitial macrophages than those in mice reconstituted with $\mathrm{Agtr} \mathrm{1}^{+/+}$marrow. These differences were not observed at day 5 of UUO. The expression of profibrotic genes - including TGF- $\beta 1, \alpha 1(\mathrm{I})$ collagen, and $\alpha 1$ (III) collagen - was substantially higher in the obstructed kidneys of mice with $\mathrm{Agtr}^{-/-}$marrow than in those with $\mathrm{Agtr} 1^{+/+}$marrow at day 14 but not at day 5 of UUO. Mice with $\mathrm{Agtr}^{-1-}$ marrow were characterized by reduced numbers of peripheral-blood monocytes and macrophage progenitors in bone marrow. In vivo assays revealed a significantly impaired phagocytic capability in $\mathrm{Agtr} \mathrm{I}^{-/-}$macrophages. In vivo treatment of $\mathrm{Agtr} \mathrm{I}^{+/+}$mice with losartan reduced phagocytic capability of $\mathrm{Agtr}^{+/+}$macrophages to a level comparable to that of Agtr1 $1^{-/-}$macrophages. Thus, during urinary tract obstruction, the Agtr1 on bone marrow-derived macrophages functions to preserve the renal parenchymal architecture, and this function depends in part on its modulatory effect on phagocytosis.
\end{abstract}

J. Clin. Invest. 110:1859-1868 (2002). doi:10.1172/JCI200215045.

\section{Introduction}

Pharmacologic blockade of angiotensin I-converting enzyme (ACE) and the angiotensin II type 1 receptor (Agtr1) has been shown to attenuate renal scarring in several experimental models and to lessen progressive loss of renal function in patients with chronic renal diseases $(1,2)$. Conversely, patients with genetic traits associated with heightened activities of the renin-angiotensin system (RAS) have been found to be at increased risk for the progression of chronic

Received for publication January 14, 2002, and accepted in revised form October 1, 2002.

Address correspondence to: Iekuni Ichikawa, MCN C4204, Department of Pediatrics, Vanderbilt University Medical Center, Nashville, Tennessee 37232-2584, USA. Phone: (615) 322-7931; Fax: (615) 322-7929; E-mail: ichikawamd@aol.com.

Conflict of interest: The authors have declared that no conflict of interest exists.

Nonstandard abbreviations used: angiotensin II type 1 receptor (Agtr1); unilateral ureteral obstruction (UUO); angiotensin I-converting enzyme (ACE); renin-angiotensin system (RAS); extracellular matrix (ECM); $\alpha$-smooth muscle actin ( $\alpha$-SMA); reverse-phase high-performance liquid chromatography (RP-HPLC); matrix metalloproteinase-2 (MMP-2); tissue inhibitor of metalloproteinase 1 (TIMP-1); colony stimulating factor 1 (CSF-1); 7-amino-actinomycin D (7-AAD); monocyte chemotactic protein 1 (MCP-1). renal diseases (3). These data suggest that the RAS is an active component of biological processes that lead to renal scarring.

Renal fibrosis has been noted to correlate with the number of bone marrow-derived macrophages within the injured renal tissue of experimental animals (4-7) and humans (8). Interventions, such as ACE inhibition, that attenuate renal fibrosis are also associated with inhibition of macrophage infiltration $(9,10)$. Shortterm studies using whole-body irradiation that eliminates macrophages find a positive correlation between the number of infiltrating macrophages and the degree of renal interstitial fibrosis (11), suggesting that macrophages promote fibrosis. However, longer-term studies (5) reveal a reciprocal relationship between these two parameters and raise questions about the function of the infiltrating cells. Recent studies of cutaneous wound healing provide some insight into these issues. Initially, a positive correlation is observed between the quantity of infiltrating macrophages and the degree of tissue fibrosis during the wound-healing process (12-15). In this setting, macrophages promote deposition of provisional extracellular matrix (ECM). However, as wound healing advances, macrophages participate in the resolution of the deposited ECM (13). 
Thus, bone marrow-derived macrophages appear to have a dual function in the fibrotic process in the skin, which may also operate in other tissues.

The fibrogenetic process in the kidney and elsewhere has been postulated to encompass contributions from both the infiltrating and resident cells. In addition to the well-documented actions of the RAS on the resident cells, there is ample in vitro evidence (16-21) indicating that bone marrow-derived macrophages express components of the RAS and respond to angiotensin II. However, it has heretofore not been possible to identify the specific function of macrophages because the existing technology did not allow separation of these two systems in vivo. Bone marrow cell transplantation and in vivo DNA recombinant technologies have become available for experimental animal studies, and these methods now allow the specific role of marrowderived cells and their specific gene products to be examined separately from those of the resident cells in vivo $(22,23)$. Since bone marrow-derived cells have also been implicated in renal scarring, we wished to evaluate the relative contribution of the infiltrating and resident cells in kidney damage. Moreover, we took advantage of the availability of cells from mice lacking a RAS gene recently generated in our laboratory (24). Using bone marrow transplantation technique and the recombinant mouse model, we ascertained the function of macrophages in renal interstitial fibrosis, with the specific focus on the role of the angiotensin II system within macrophages.

\section{Methods}

Mice. Agtr1 $1^{-/-}$donor mice used in the present study were generated in our laboratory (24) and had been backcrossed more than 10 times with C57BL/6 mice (The Jackson Laboratory, Bar Harbor, Maine, USA). Thus, all mice used in the study (both the genetically manipulated donors and the wild-type recipient mice) were on the C57BL/6 background. Rodents carry two subtypes of the Agtr 1 receptor - namely, type $1 \mathrm{a}$ and type $1 \mathrm{~b}$ (25). Previous data have demonstrated that the angiotensin II type 1 a receptor is by far the predominant isoform in mice (26). We therefore used the angiotensin II type 1a receptor null mutant mice, designated as Agtr1 $1^{-/}$mice, in the present study. The genotype of these mice was determined by Southern blot analysis on DNA isolated from tail biopsies as previously described (24). Male C57BL/6 mice were purchased from The Jackson Laboratory.

Bone marrow reconstitution and unilateral ureteral obstruction. Bone marrow transplantation was performed as previously described $(22,23)$. Three-month-old male recipient mice were lethally irradiated with 9 Gy using a cesium gamma source. Bone marrow cells were harvested from the femurs and tibias of donor mice by flushing with RPMI-1640 media (Gibco BRL, Gaithersburg, Maryland, USA), and recipients received $5 \times 10^{6}$ bone marrow cells per mouse in $0.2 \mathrm{ml}$ of media by tail-vein injection. To verify the reconstitution of bone marrow after transplantation, the bone marrow and peritoneal macrophages were collected at the time of sacrifice, genomic DNA was extracted from these cells, and the genotype was determined by Southern blot analysis as we have previously described (24).

Eight weeks after bone marrow transplantation, the transplanted mice were subjected to a complete unilateral ureteral obstruction (UUO) as previously described (27). Briefly, under sodium pentobarbital anesthesia, the middle portion of the left ureter was ligated and cut between the two ligated points. At 5 and 14 days after surgery, the mice were sacrificed, and the obstructed kidneys were harvested and subjected to the studies described below. The experimental protocols were performed according to the regulations of the Vanderbilt University Animal Care Committee.

Histological study. For histological examinations, kidneys were fixed with $4 \%$ buffered paraformaldehyde for 6 hours, embedded in paraffin, and sectioned transversely with a thickness of $4 \mu \mathrm{m}$. A standard pointcounting method was used to quantitate the collagen fractional volume in the renal cortical interstitium on Masson trichrome-stained sections (magnification, $\times 400$ ), as described in our previous studies (27). The index of interstitial collagen fractional volume was defined as the number of trichrome-positive points in every 1000 points evaluated. For immunohistochemistry, monoclonal rat anti-mouse F4/80 (Selotec, Oxford, United Kingdom) and anti-mouse CD3- $\varepsilon$ (clone 48-2B; Santa Cruz Biotechnologies Inc., Santa Cruz, California, USA) were used to detect infiltrating macrophages and $\mathrm{T}$ cells, respectively $(27,28)$. $\alpha$-Smooth muscle actin ( $\alpha$-SMA) was stained by mouse monoclonal anti-human $\alpha$-SMA (clone 1A4; DAKO, Carpinteria, California, USA) $(27,28)$. Vascular endothelial cells were stained with a monoclonal rat antibody to a cell membrane antigen present on endothelial cells, CD31 (clone MEC 13.3, 1:50 dilution; Pharmingen, San Diego, California, USA) (29). After exposure to $0.1 \%$ trypsin (DAKO) for 10-15 minutes and quenching with $3 \%$ hydrogen peroxide in methanol, sections were incubated with rat antimouse CD31 for 1 hour at room temperature, followed by standard $\mathrm{ABC}$ immunostaining.

Macrophage infiltration was determined by enumerating F4/80-positive cells within the cortical interstitium in 10 randomly selected cortical fields under magnification ( $\times 400$ ), and the numbers were averaged for each field (27). The density of CD31-positive peritubular capillary was quantified as the peritubular capillary rarefaction index as previously described (30). Briefly, the squares in a $10 \times 10$ grid that showed no capillaries were counted in 10 sequential fields under magnification $(\times 200)$ and averaged. The expression of $\alpha$-SMA in the cortical interstitial area was assessed semiquantitatively by immunoreactivity for $\alpha$-SMA as previously described (28).

Collagen content. The collagen content of renal cortex was calculated from the concentrations of hydroxyproline and proline, which were determined through 
measurement of their phenylisothiocyanate derivatives by reverse-phase high-performance liquid chromatography (RP-HPLC) as previously described (31).

Northern blot analyses for TGF- $\beta 1, \alpha 1(I)$ collagen, $\alpha 1(I I I)$ collagen, matrix metalloproteinase 2 , and tissue inhibitor of metalloproteinase 1 mRNA. Total RNA was extracted by the RNA STAT-60 method (Tel-Test Inc., Friendswood, Texas, USA), electrophoresed, transferred to a nylon membrane (Hybond- $\mathrm{N}^{+}$; Amersham Life Science Inc., Arlington Heights, Illinois, USA), and hybridized with a ${ }^{32} \mathrm{P}-$ labeled probe. The cDNA probes for mouse TGF- $\beta 1, \alpha 1$ (I) collagen, $\alpha 1$ (III) collagen, matrix metalloproteinase-2 (MMP-2), and tissue inhibitor of metalloproteinase 1 (TIMP-1) were used as described previously $(27,28)$. The hybridization signal of each mRNA was detected by autoradiography, scanned with a JX-330M scanner (Sharp Co., Tokyo, Japan), and semiquantitated using the National Institutes of Health image program.

Hematologic analysis. Total leukocyte, red blood cell, and platelet counts were determined on peripheral blood collected by retro-orbital venous plexus puncture using an ADVIA 120 automated counter (Bayer, Tarrytown, New York, USA), and the manual 100-cell leukocyte differential counting was performed on MayGrüwald/Giemsa-stained smears.

Determination of bone marrow macrophage progenitors. Macrophage progenitors present in the bone marrow were determined as granulocyte or macrophage colony-forming unit by semisolid agar culture (32). Bone marrow cells were harvested by flushing the tibia with RPMI-1640 media and were cultured for 10 days at a density of 50,000 cells per milliliter in media consisting of RPMI-1640, $0.3 \%$ agar, $10 \%$ FBS, and recombinant mouse colony stimulating factor 1 (CSF-1) at concentrations indicated in Figure 6. Macrophage and granulocyte/macrophage colonies containing more than 50 cells were morphologically identified in triplicate cultures, counted, and expressed as colony-forming units per tibia.

Determination of macrophage migrating activity. Peritoneal macrophages were collected from mice 4 days after peritoneal injection of $3 \%$ thioglycollate. Chemotaxis was performed in a 96-well microchemotaxis chamber in which the upper compartment was separated from the lower compartment by a single uncoated polycarbonate filter $(5-\mu \mathrm{m}$ pore diameter; Neuroprobe, Cabin John, Maryland, USA). Peritoneal macrophages were suspended in DMEM without FBS and added to the upper compartment of the chemotaxis chamber at a density of $1.2 \times 10^{6}$ cells per well. Chemoattractants were added to the lower compartment, and chambers containing cells and chemoattractants were incubated at $37^{\circ} \mathrm{C}$ for 2.5 hours in an atmosphere of $95 \%$ air, $5 \% \mathrm{CO}_{2}$, and $100 \%$ humidity. After incubation, cells remaining on the upper surface of the filter were removed mechanically. Filters were then fixed in methanol and stained with $1 \%$ crystal violet. Migrated cells adhering to the lower surface of the membrane were counted manually under the microscope with an oil-immersion lens $(\times 400)$. Triplicate wells were used for each experimental condition, and 10 fields were examined for each well.

Measurement of Fcyreceptor-mediated oxidative burst. Fcy receptor-mediated oxidant production in peritoneal macrophages was measured using the FcOxyBurst reagent (Molecular Probes, Eugene, Oregon, USA) according to the manufacturer's instructions. Briefly, peritoneal macrophages harvested from mice 4 days after peritoneal injection of $3 \%$ thioglycollate were suspended at $2 \times 10^{6}$ cells per milliliter in PBS supplemented with $1 \mathrm{~g} / 1$ glucose and then stimulated with FcOxyBurst at a final concentration of $140 \mu \mathrm{g} / \mathrm{ml}$. Flow cytometric analysis was performed with a FACScan flow cytometer (Becton Dickinson, Franklin Lakes, New Jersey, USA) gating to select F4/80-positive macrophages. Twenty thousand 7-amino-actinomycin $\mathrm{D}$-negative (7-AAD-negative), living cells were obtained for the analysis.

In vivo and in vitro assays of phagocytic activity of macrophages. Phagocytic activity of macrophages in vivo was examined using previously described methods (33, 34). Four days after thioglycollate injection, $2.5 \mathrm{ml}$ of a $0.2 \%$ suspension of Fluoresbrite fluorescent microspheres (0.75 YG; Polyscience, Warrington, Pennsylvania, USA) was injected into the peritoneal cavity. After 1 hour of incubation, peritoneal fluid containing cells and fluorescent beads was collected. The macrophages with fluorescent beads were counted in accordance with the amount of ingested beads (34), and the nonphagocytosed free beads were also quantified by flow cytometry as a percentage of the total number of beads (33) on a FACScan flow cytometer using CellQuest software (Becton Dickinson) for data acquisition and Winlist software (Verity Software House, Topsham, Maine, USA) for data analysis. Twenty thousand 7-AAD-negative, living cells were obtained for the analysis. The flow cytometric analysis was performed gating to select F4/80-positive macrophages, and the fluorescence of extracellular beads was quenched using crystal violet according to the manufacturer's protocol. To determine the role of Agtr 1 in vivo, losartan was added to the drinking water $(200 \mathrm{mg} / \mathrm{l}) 18$ hours before the assay, and a bolus of $1.0 \mathrm{mg}$ was also injected into the peritoneal cavity with fluorescent beads.

For the in vitro phagocytic assay, peritoneal macrophages were collected from mice 4 days after peritoneal injection of $3 \%$ thioglycollate, and approximately $2 \times 10^{6}$ peritoneal macrophages on 24-well plates in DMEM with $10 \%$ FBS were used $(33,35)$. One hundred microliters of $0.26 \%$ latex solution of Fluoresbrite fluorescent microspheres ( 0.75 YG; Polyscience) was added to each well and incubated for 30 minutes at $37^{\circ} \mathrm{C}$. Nonadherent latex beads were removed by washing, and the cells were incubated for another 30 minutes to allow for complete phagocytosis. Cells were then harvested by treatment with PBS containing $5 \mathrm{mM}$ EDTA and washed vigorously three 


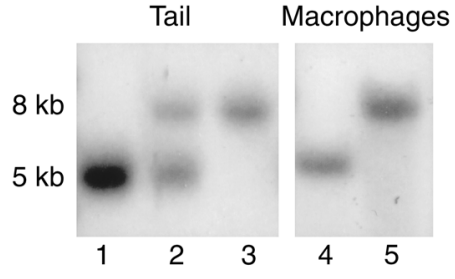

Figure 1

Southern blot genotype analysis of macrophages isolated from mice transplanted with either Agtr1 $1^{+/+}$or Agtr1 ${ }^{-/-}$bone marrow. Lanes 1-3 show DNA isolated from tail biopsy specimens of Agtr1-/- mice (Lane 1), Agtr $1^{+/-}$mice (Lane 2), and Agtr1 $1^{+/+}$mice (Lane 3). Lanes 4 and 5 show DNA isolated from peritoneal macrophages of wild-type mice reconstituted with Agtr1 ${ }^{-/-}$marrow (Lane 4) and wild-type mice with Agtr $1^{+/+}$marrow (Lane 5). The DNA in lane 4 represents pooled macrophage DNA from 10 mice reconstituted with Agtr1-/- marrow.

times with PBS to remove nonphagocytosed beads. The amount of phagocytosed beads was measured by flow cytometry as described above. In some experiments, either the Agtr1 antagonist losartan or angiotensin II (Bachem, Torrance, California, USA) was added to the final concentration of $10^{-6} \mathrm{M} 18$ hours or 3 hours before the phagocytosis assay, respectively. The amount of nonspecifically bound beads was determined by incubating macrophages with fluorescent beads on ice.

Statistical analysis. Data are presented as means \pm SEM. Statistical analysis was performed by ANOVA, and statistical significance was defined as $P<0.05$.

\section{Results}

Reconstitution of Agtr $1^{+/+}$mice with Agtr1 $1^{-/-}$bone marrow. Resident macrophages of the recipient mice were removed from the peritoneal cavity at the time of sacrifice and genotyped (Figure 1). Uniformly, only the $5-\mathrm{kb}$ band was observed in the recipient mice, demonstrating that $\mathrm{Agtr}^{+/+}$mice were successfully reconstituted with $\mathrm{Agtr}^{-1}$ marrow, and macrophages were primarily from the donor (lane 4 in Figure 1). This finding is in good agreement with our previous bone marrow reconstitutions (22).

Effect of Agtr1 $1^{-/}$bone marrow transplantation on interstitial macrophage infiltration in the obstructed kidneys. Macrophage accumulation in the kidney was examined in wild-type mice with $A g t r 1^{-/-}$marrow and wild-type mice with Agtr $1^{+/+}$marrow at day $5\left(\right.$ Agtr1 $^{-/-}$marrow, $n=9 ; \mathrm{Agtr}^{+/+}$ marrow, $n=10)$ and day $14\left(\right.$ Agtr $1^{-/-}$marrow, $n=8$; Agtr1 $1^{+/}$marrow, $n=10$ ) after surgical creation of UUO. At day 5, there was no appreciable difference in the number of interstitial macrophages between the two groups $(17.3 \pm 2.5$ vs. $19.7 \pm 3.0$, not significant) (Figure $2 b)$. At 14 days after UUO, mice carrying transplanted Agtr $1^{-/}$ marrow showed significantly lower numbers of F4/80positive interstitial macrophages than those carrying Agtr $1^{+/+}$marrow (27.6 \pm 1.5 vs. $\left.41.8 \pm 2.5, P<0.001\right)$ (Figure 2 , $a$ and $b$ ). Staining for $\mathrm{CD} 3$ revealed that there were only a few $\mathrm{T}$ cells in the interstitium in both groups at either day 5 or day 14 after UUO. No significant difference was observed between the groups (data not shown).
Effect of Agtr1 1-/- bone marrow transplantation on renal fibrosis in the obstructed kidneys. The degree of fibrosis was compared between wild-type mice with Agtr1 $1^{-/-}$marrow or $\mathrm{Agtr}^{+/+}$marrow at day 5 and day 14 after UUO (Figure 3a). At day 5 after UUO, there was no difference in interstitial fibrosis ( $44 \pm 8$ vs. $42 \pm 6$ points per 1000 points, not significant) (Figure 4a). However, at 14 days after UUO, mice carrying Agtr1 ${ }^{-/-}$marrow $(n=8)$ showed significantly more severe interstitial fibrosis than those carrying Agtr $1^{+/+}$marrow $(n=10 ; 139 \pm 9$ vs. $93 \pm 9$ points per 1000 points, $P<0.005$ ) (Figure 4a). The enhanced fibrosis seen in mice carrying $\mathrm{Agtr}^{-/-}$ marrow was confirmed independently by a biochemical determination of collagen content. Thus, collagen content in renal tissue assessed by RP-HPLC was also significantly higher in mice with Agtr1 $1^{-/-}$marrow than in mice with Agtr $^{+/+}$marrow $(11.6 \% \pm 1.1 \%$ vs. $7.7 \% \pm 0.5 \%$ collagen, $P<0.005)$ (Figure 4b).

Using CD31 staining, we also evaluated peritubular capillary density. The new generation of peritubular capillaries is believed to be promoted by macrophagederived bFGF in early stages of UUO (36). No significant difference in the peritubular capillary density was seen between the two groups 5 days after UUO (rarefaction index, $4.3 \% \pm 0.5 \%$ vs. $4.9 \% \pm 0.7 \%$, not significant) (Figure 3b). Transdifferentiation of tubular epithelial cells to myofibroblasts has been implicated as another component of the fibrogenetic processes in UUO (37). Cells with $\alpha$-SMA-positive staining were located in the renal interstitium and vessel walls (Figure 3c). We found no significant

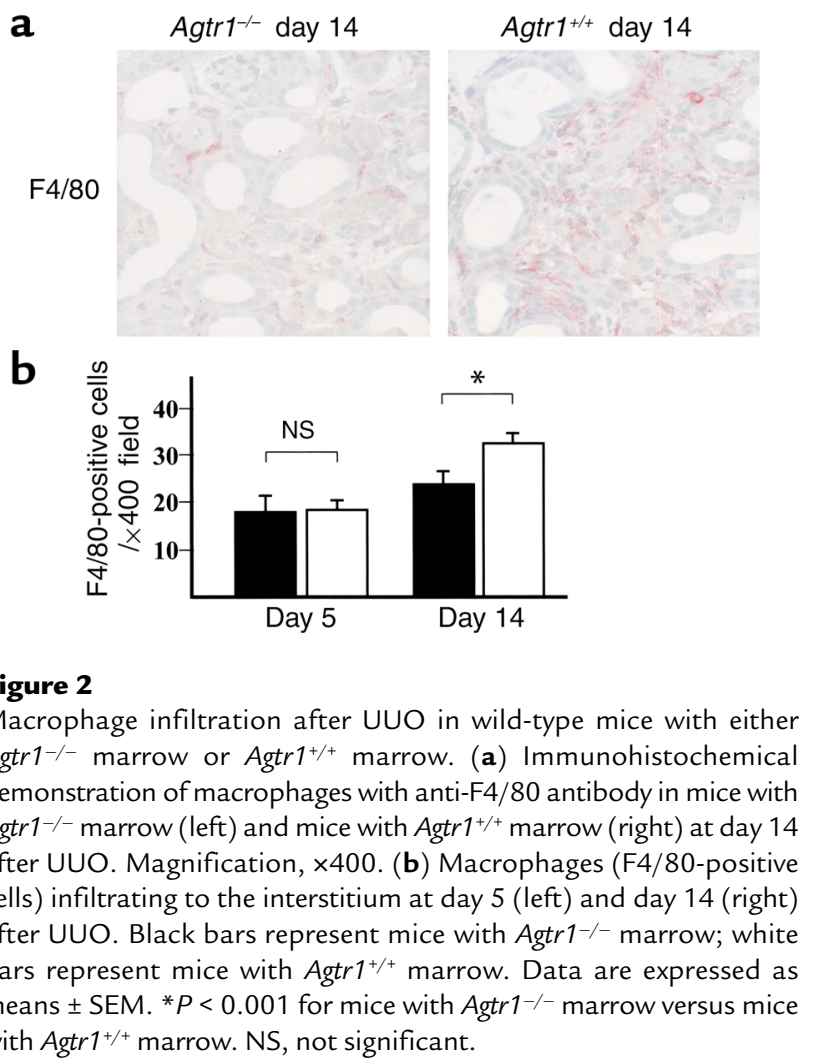



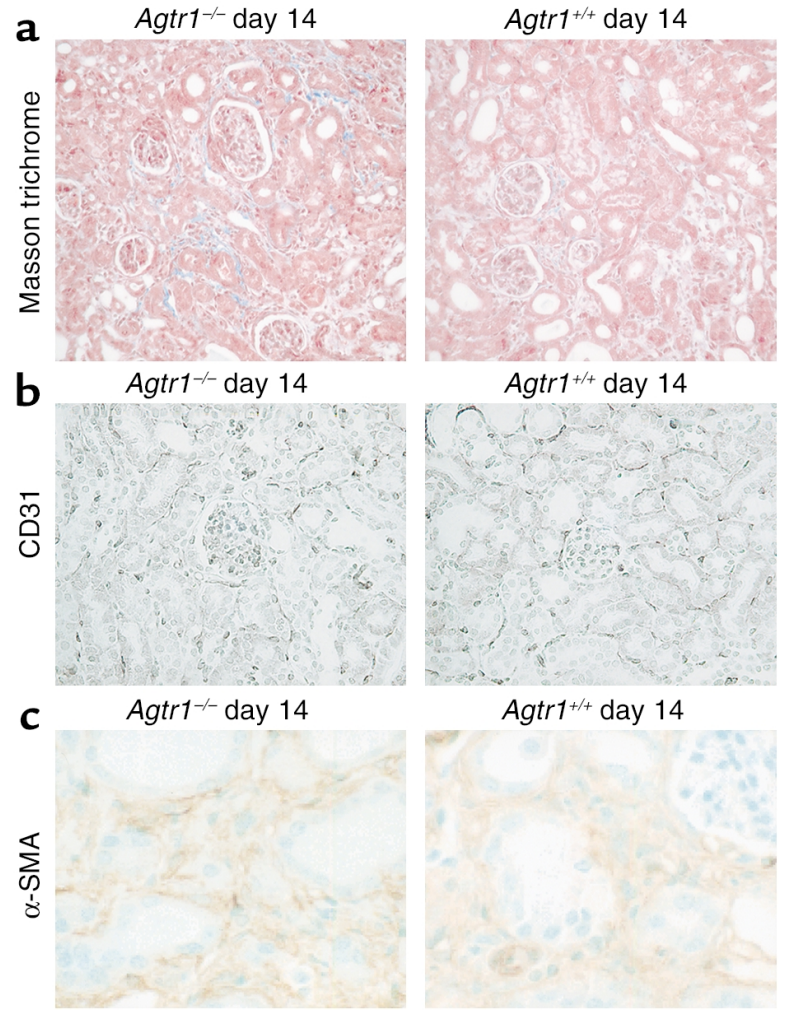

Agtr1 $^{+/+}$day 14

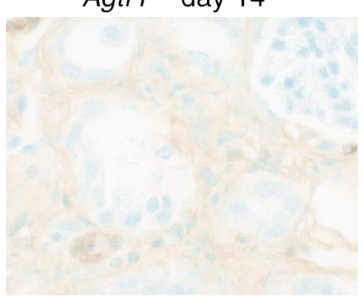

\section{Figure 3}

Histological assessment of interstitial fibrosis and peritubular capillary density and detection of myofibroblasts in the obstructed kidneys of wild-type mice with either Agtr $1^{-/-}$marrow or Agtr1 $1^{+/+}$marrow. Masson trichrome staining of the renal cortex at day 14 after UUO (a), immunohistochemical assessment of peritubular capillary density by immunostaining with anti-CD31 antibody at day 5 after UUO (b), and detection of myofibroblasts by immunostaining with anti$\alpha S M A$ antibody at day 14 after $U \cup O(c)$ in mice with Agtr $1^{-/-}$marrow (left) and mice with Agtr $1^{+/+}$marrow (right). Magnification, $\times 400$.

difference in the immunoreactivity of $\alpha$-SMA between the two groups 14 days after UUO ( $\alpha$-SMA score, $2.66 \pm 0.06$ vs. $2.44 \pm 0.10$, not significant).

$m R N A$ expressions for molecules involved in the accumulation of ECM. In parallel with the pattern of interstitial fibrosis, expression of TGF- $\beta 1 \mathrm{mRNA}$ was significantly higher in mice with ggtr $^{-/-}$marrow than in mice with Agtr1 $1^{+/}$ marrow at day 14 after UUO (the ratio of optical density to that of GAPDH mRNA, $2.08 \pm 0.23$ vs. $0.59 \pm 0.08$, $P<0.001)$. TGF- $\beta 1$ mRNA expression was similar at day $5(1.07 \pm 0.12$ vs. $1.19 \pm 0.09$, not significant) (Figure 5a). In mice with Agtr $1^{+/+}$marrow, TGF- $\beta 1$ mRNA expression was not significantly different between day 5 and day 14 . In contrast, in mice with Agtr $1^{-/}$marrow, TGF- $\beta 1$ mRNA expression was significantly increased by day 14 from the level seen on day 5 (Figure 5a).

Figure 5 (b and c) summarizes mRNA expression for molecules involved in accumulation as well as those involved in degradation of ECM. Profibrotic molecules, including $\alpha 1$ (I) collagen and $\alpha 1$ (III) collagen, were expressed at substantially higher levels in mice with Agtr1 $1^{-/-}$marrow than in mice with Agtr $1^{+/+}$marrow at day $14(\alpha 1[\mathrm{I}]$ collagen: $1.93 \pm 0.39$ vs. $0.85 \pm 0.14$, $P<0.05$; $\alpha 1$ [III] collagen: $2.79 \pm 0.61$ vs. $1.25 \pm 0.11$, $P<0.05)$. Likewise, the expression of MMP-2 mRNA was higher in mice with Agtr1 ${ }^{-/-}$marrow than those with Agtr $^{+/+}$marrow $(2.40 \pm 0.43$ vs. $0.74 \pm 0.24$, $P<0.01$ ), whereas no significant difference was observed in the expression of TIMP- 1 mRNA at day 14 after UUO ( $1.20 \pm 0.36$ vs. $1.83 \pm 0.20)$ (Figure 5c). At day 5 after UUO, no significant difference was observed between mice with Agtr1 $1^{-/-}$marrow or Agtr1 $1^{+/+}$marrow in $\alpha 1$ (I) collagen mRNA $(1.27 \pm 0.14$ vs. $1.38 \pm 0.13)$, $\alpha 1$ (III) collagen mRNA (0.60 \pm 0.08 vs. $0.49 \pm 0.09)$, MMP- 2 mRNA $(0.98 \pm 0.11$ vs. $1.00 \pm 0.12)$, or TIMP- 1 mRNA $(1.57 \pm 0.16$ vs. $1.71 \pm 0.18)$ (Figure $5 b$ ).

Peripheral monocytes and bone marrow macrophage progenitors. Interstitial macrophages are the major infiltrating cells in UUO and are believed to be an active element of the interstitial fibrosis. Therefore, to unveil the mechanisms underlying the reduced macrophage infiltration and the enhanced fibrosis seen in wild-type mice with Agtr1 ${ }^{-/}$marrow, we examined a few features of macrophages. The number of circulating hematopoietic cells was examined in wild-type mice with Agtr1 $1^{-/-}$marrow or Agtr1 $1^{+/+}$marrow 8 weeks after bone marrow transplantation (Table 1). Leukocytes were significantly less abundant in mice with Agtr1 $1^{-/-}$marrow than in those with Agtr1 $1^{+/+}$marrow. Although the number of monocytes in mice with Agtr1 $1^{-/}$marrow was significantly lower than in those with Agtr $^{+/+}$marrow, the monocyte/white blood cell ratio was similar between the two groups of mice. Thus, the difference is not unique to monocytes.

Total bone marrow cells per tibia were not significantly different between mice with Agtr $^{+/+}$or Agtr1 $^{-/-}$ marrow $\left(22.3 \pm 4.1 \times 10^{6}\right.$ cells per tibia vs. $17.6 \pm 3.8 \times 10^{6}$ cells per tibia, not significant). When 50,000 marrow cells were cultured for 10 days in semisolid agar culture in the presence of increasing concentrations of cerebrospinal fluid 1 (CSF-1), growth of macrophage progenitor colonies reached a plateau at $5 \mathrm{ng} / \mathrm{ml}$ of CSF-1 for both $\mathrm{Agtr}^{+/+}$and Agtr1 ${ }^{-/-}$marrow (Figure 6a). Thus,
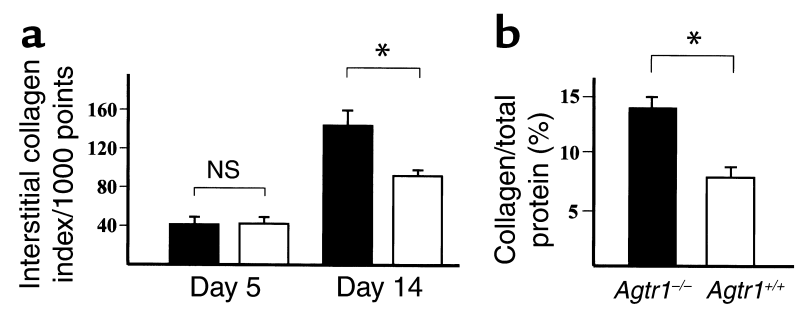

\section{Figure 4}

Quantitative analyses of interstitial collagens after UUO in wild-type mice with either Agtr1 $1^{-/-}$marrow or Agtr $1^{+/+}$marrow. (a) Interstitial collagen index assessed by point-counting method on Masson trichrome-stained sections at day 5 (left) and day 14 (right) after UUO. (b) Collagen content as percentage of total protein in kidney at day 14 after UUO. Black bars represent mice with Agtr1-/- marrow; white bars represent mice with Agtr1 ${ }^{+/+}$marrow. Data are expressed as means \pm SEM. ${ }^{*} P<0.005$ for mice with Agtr $1^{-1-}$ marrow versus mice with $A g t r 1^{+/+}$marrow. 
a
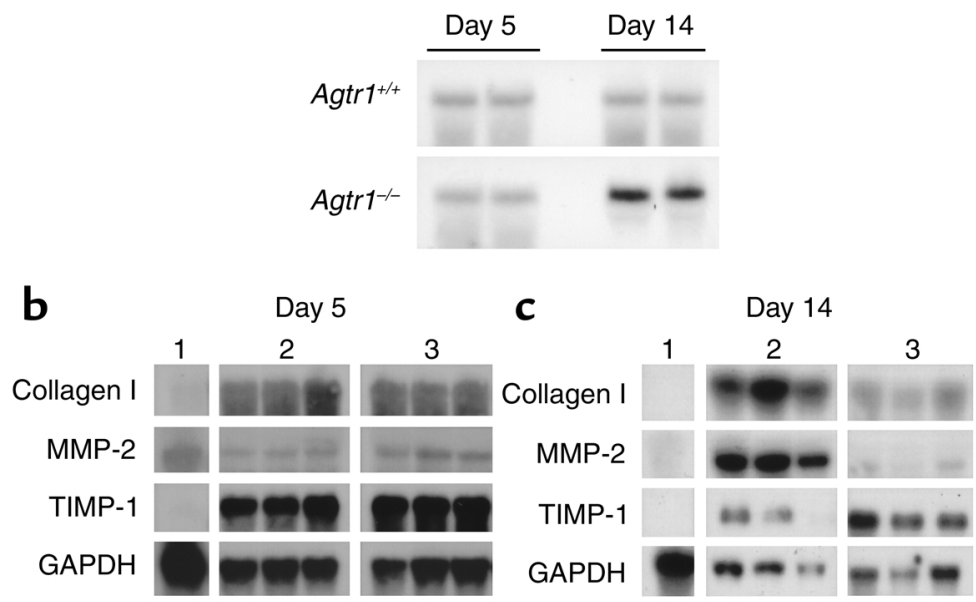

\section{Figure 5}

mRNA expressions of molecules involved in the accumulation of ECM. (a) Northern blot analyses of TGF- $\beta 1$ expressions at day 5 (left) and day 14 (right) after UUO in wild-type mice with Agtr1-/marrow and wild-type mice with Agtr $1^{+/+}$marrow. (b and c) Northern blot analyses of $\alpha 1$ (I) collagen, MMP-2, and TIMP-1 at day 5 (b) and day 14 (c) after UUO. Lane 1 in (b) and (c) shows control contralateral nonobstructed kidney, lane 2 shows mice with Agtr1-/- marrow, and lane 3 shows mice with Agtr $1^{+/+}$marrow. this assay showed that mice with $A g t r 1^{-/-}$marrow had significantly less macrophage progenitors than those with Agtr $1^{+/+}$marrow $\left(18.0 \pm 2.8 \times 10^{3}\right.$ cells per tibia vs. $25.5 \pm 3.2 \times 10^{3}$ cells per tibia, $P<0.05$ ) (Figure $6 \mathrm{~b}$ ).

Functions of macrophages. To explore further the mechanisms responsible for the enhanced fibrosis in mice with Agtr1 $1^{-/-}$marrow, we next examined macrophage functions. Monocyte chemotactic protein 1 (MCP-1) has been shown to be the major chemotactic factor responsible for macrophage infiltration in UUO (38), and chemotactic activity of angiotensin II has been demonstrated for human monocytes (39). On stimulation with $0.1 \mu \mathrm{g} / \mathrm{ml} \mathrm{MCP}-1, \operatorname{Agtr} 1^{+/+}$macrophages exhibited a maximal 1.5 -fold increase in chemotactic response from $124 \pm 6$ cells per field to $186 \pm 41$ cells per field (left panel in Figure $7 \mathrm{a}$ ). In response to 0.1 $\mu \mathrm{g} / \mathrm{ml}$ MCP-1, a similar 1.5-fold increase was observed in the chemotactic response of Agtr $1^{-/-}$macrophages from $34 \pm 7$ cells per field to $51 \pm 11$ cells per field, indicating that although the baseline motility of Agtr1-/macrophages is lower than that of $A g t r 1^{+/+}$macrophages, macrophages of either genotype have indistinguishable chemotactic responses to MCP-1. Addition of $1 \times 10^{-6} \mathrm{M}$ angiotensin II, either alone or with 0.1 $\mu \mathrm{g} / \mathrm{ml}$ MCP-1, was without effect on the motility of Agtr $1^{+/+}$macrophages (right panel in Figure 7a).

Reactive oxygen species are potent destructive effector molecules produced by macrophages and are thought to be involved in the renal damage occurring in UUO (40). On stimulation through the Fc $\gamma$ receptor, Agtr1 $1^{+/}$and Agtr1 $1^{-/}$macrophages showed similar oxidative burst (mean fluorescence intensity of total macrophages after 15 minutes of incubation, $35.8 \pm 0.9$ vs. $38.1 \pm 0.9$, not significant) (Figure $7 \mathrm{~b}$ ), suggesting that together with reduced macrophage infiltration, macrophage-mediated oxidative injury would be less significant in mice with Agtr $1^{-/-}$macrophages than in those with $A g t r 1^{+/+}$macrophages.

Active removal of ECM has been suggested as a critical element that maintains the dynamic balance of the highly regulated wound-healing process, and macrophages are thought to participate in ECM removal through phagocytosis (13). To examine the phagocytic capability of macrophages in vivo, we injected fluorescent beads into the peritoneal cavity after inducing macrophages with thioglycollate. Analysis of the peritoneal fluid obtained 1 hour later showed that both the percentage of macrophages with phagocytosed beads and the mean fluorescence intensity were greater in $A g t r 1^{+/+}$mice than in $A g t r 1^{-/-}$mice $(85.6 \% \pm 1.2 \%$ vs. $49.8 \% \pm 1.4 \%$ phagocytic macrophages, $P<0.001$; mean fluorescence intensity of total macrophages, $208.6 \pm 12.7$ vs. $109.4 \pm 8.3, P<0.005)$ (Figure 8a). Similarly, fewer fluorescent beads were free (nonphagocytosed) in Agtr1 $1^{+/+}$than in Agtr $1^{-/-}$mice (remaining free beads, $56.8 \% \pm 6.8 \%$ vs. $81.8 \% \pm 3.5 \%$, $P<0.005$; duplicated experiments, $n=6$ per group). The effect of losartan on the phagocytic capability of Agtr $1^{+/+}$macrophages was also examined in vivo. The losartan treatment caused a significant decrease in the phagocytic capability of Agtr $1^{+/+}$macrophages in vivo $(85.6 \% \pm 1.2 \%$ vs. $47.3 \% \pm 2.2 \%$ phagocytic macrophages, $P<0.001$; mean fluorescence intensity of total macrophages, $208.6 \pm 12.7$ vs. $99.1 \pm 5.1, P<0.005$; remaining free beads, $56.8 \% \pm 6.8 \%$ vs. $82.9 \% \pm 4.8 \%$, $P<0.05$ ) (Figure $8 \mathrm{a}$ ). Thus, the phagocytic capability

Table 1

Number of circulating blood cells in mice with Agtr1 ${ }^{-/-}$and Agtr1 ${ }^{+/+}$marrow

\begin{tabular}{lccccccc}
\hline Donor & $n$ & $\mathrm{WBC} / \mu \mathrm{l}$ & $\mathrm{RBC} \times 10^{4} / \mu \mathrm{l}$ & $\mathrm{PLT} \times 10^{4} / \mu \mathrm{l}$ & Monocyte/WBC (\%) & Lymphocyte/WBC (\%) & Neutrophil/WBC (\%) \\
Agtr1 $1^{-/}$ & 10 & $6799 \pm 546^{\mathrm{A}}$ & $1008 \pm 12$ & $99 \pm 3$ & $12 \pm 2$ & $80 \pm 2$ & $1.5 \pm 0.2$ \\
Agtr1 $^{+/+}$ & 9 & $8556 \pm 537$ & $1007 \pm 18$ & $91 \pm 5$ & $13 \pm 3$ & $80 \pm 3$ & $1.7 \pm 0.2$
\end{tabular}

WBC, white blood cells; RBC, red blood cells; PLT, platelets. Data are expressed as means \pm SEM. ${ }^{A} P<0.05$ versus Agtr $1^{+/+}$. 

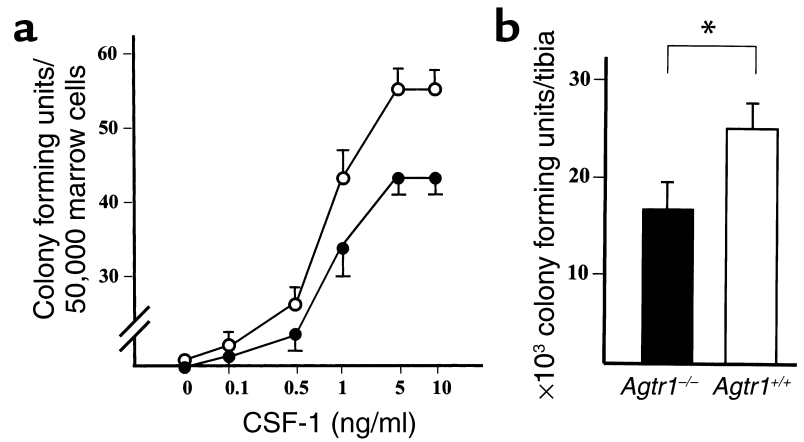

\section{Figure 6}

Macrophage progenitors in bone marrow. Bone marrow cells isolated from either Agtr1 $1^{+/+}$mice or Agtr1 $1^{-/-}$mice were cultured for 10 days in semisolid agar culture at a density of 50,000 cells per milliliter in the presence of indicated concentrations of CSF-1. Colonies of macrophages or granulocyte/macrophages were counted (a), and the total number of macrophage progenitors per tibia was determined (b). ${ }^{*} P<0.05$.

of $A g t r 1^{-/-}$macrophages is reduced in vivo. Furthermore, the Agtr1 antagonist losartan decreased the phagocytic activity of $A g t r 1^{+/+}$macrophages in vivo to a level comparable to that of Agtr $1^{-/-}$macrophages.

In the in vitro phagocytosis assay, isolated macrophages were incubated with fluorescent beads, and the macrophages with fluorescent beads were counted by flow cytometry in accordance with the amount of ingested beads $(33,35)$. Representative results are shown in Figure 8b. There was no difference in the number of macrophages with beads (phagocytic macrophages) between Agtr1 ${ }^{+/+}$and Agtr1 $1^{-/-}$macrophages $(84.9 \% \pm 3.0 \%$ vs. $79.4 \% \pm 2.6 \%$ phagocytic macrophages, not significant; duplicate experiments, $n=6$ per group). By contrast, there were significantly more beads in $\mathrm{Agtr}^{+/+}$macrophages than in $\mathrm{Agtr1}^{-/}$ macrophages (mean fluorescence intensity of total macrophages, $722.3 \pm 53.9$ vs. $498.4 \pm 21.9, P<0.005$ ). There was no difference between Agtr $1^{+/+}$and Agtr1 $1^{-/-}$ macrophages in the number of macrophages with nonspecifically bound beads ( $8.9 \%$ of Agtr $1^{+/+}$macrophages and $10.4 \%$ of $\mathrm{Agtr1}^{-/-}$macrophages) or in the fluorescence intensity of nonspecifically bound beads (mean fluorescence intensity of total macrophages; $11.5 \mathrm{vs}$. 12.7, respectively). The Agtr1 antagonist losartan had no significant effect on either the number of the phagocytic macrophages $(84.9 \% \pm 3.0 \%$ vs. $79.5 \% \pm 0.8 \%$, not significant) or the number of phagocytosed beads (mean fluorescence intensity of total macrophages, $722.3 \pm 53.9$ vs. $642.0 \pm 30.4$, not significant). No significant difference was also observed between Agtr $1^{+/+}$ macrophages with or without angiotensin II treatment at $10^{-6} \mathrm{M}(86.4 \% \pm 1.2 \%$ vs. $84.9 \% \pm 3.0 \%$ phagocytic macrophages, not significant; mean fluorescence intensity of total macrophages, $722.3 \pm 53.9$ vs. $736.1 \pm 35.8$, not significant).

Together, these in vivo and in vitro analyses indicate that $\mathrm{Agtr}^{\mathrm{I}^{++}}$macrophages are exposed to angiotensin
II in vivo, exhibit elevated phagocytic capability, and, as a consequence, are not fully susceptible to either deactivation by losartan or further activation by angiotensin II in vitro.

\section{Discussion}

In the present study, we demonstrated that the Agtr1 genotype of bone marrow cells has a profound effect on the renal response to injury in mouse UUO. When the bone marrow was transplanted from Agtr1 ${ }^{-/-}$into Agtr1 ${ }^{+/+}$wild-type mice, the accumulation of macrophages in the kidney occurring in response to an obstructive insult was substantially attenuated, whereas renal interstitial fibrosis and profibrotic activity were enhanced. This divergence between macrophage accumulation and fibrosis was evident in late stages (day 14) but not in early stages (day 5) of UUO. Since infiltrating macrophages are thought to be a critical determinant of fibrogenesis, these data suggest that the Agtr1 on bone marrow-derived macrophages promotes preservation of the renal parenchyma during the evolution of renal fibrosis in obstructive nephropathy.

Southern blot analyses of peritoneal macrophages showed that 8 weeks after the bone marrow transplantation, macrophages were successfully repopulated by Agtr1 $1^{-/}$cells (Figure 1). On induction of UUO in wildtype mice reconstituted with either Agtr1 ${ }^{+/+}$or Agtr1 $1^{-/-}$ marrow, both fibrogenetic processes and macrophage infiltration proceeded similarly until day 5 after the a

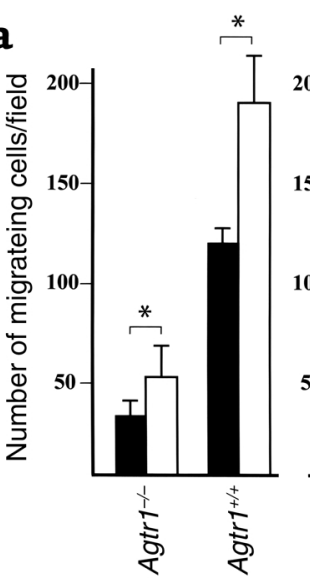

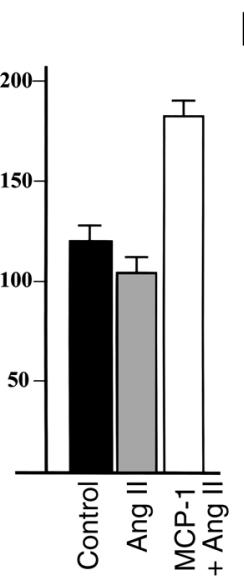

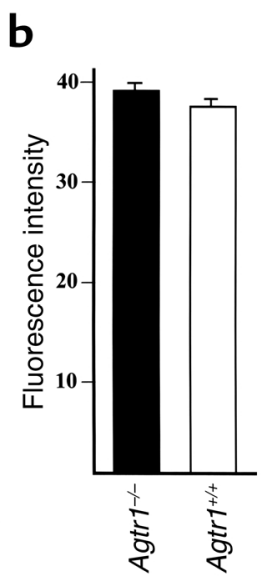

\section{Figure 7}

Migrating activity and oxidative burst of Agtr1-1- and Agtr1 ${ }^{+/+}$ macrophages. (a) Migrating activity of isolated peritoneal macrophages was determined by using chemotaxis chambers. In the left panel, Agtr1 ${ }^{-/-}$or Agtr $1^{+/+}$macrophages were incubated with either vehicle (black bars) or $0.1 \mu \mathrm{g} / \mathrm{ml} \mathrm{MCP-1(white} \mathrm{bars).} \mathrm{In} \mathrm{the} \mathrm{right} \mathrm{panel,}$ Agtr $1^{+/+}$macrophages were exposed to vehicle (white bar), $1 \times 10^{-6} \mathrm{M}$ angiotensin II alone (gray bar), or $0.1 \mu \mathrm{g} / \mathrm{ml} \mathrm{MCP}-1$ and $1 \times 10^{-6} \mathrm{M}$ angiotensin II (black bar). Migrated cells were counted and are shown as the number of migrated cells per field. ${ }^{*} P<0.05$ for vehicle versus MCP-1. (b) Macrophages of the Agtr1-/- (black bar) or the Agtr1 ${ }^{+/+}$ (white bar) genotype were examined for $\mathrm{Fc} \gamma$ receptor-mediated oxidative burst. Oxidative burst was detected as fluorescence emitted by macrophages and is indicated in arbitrary units. Ang II, angiotensin II; MCP-1, monocyte chemotactic protein-1. 
a
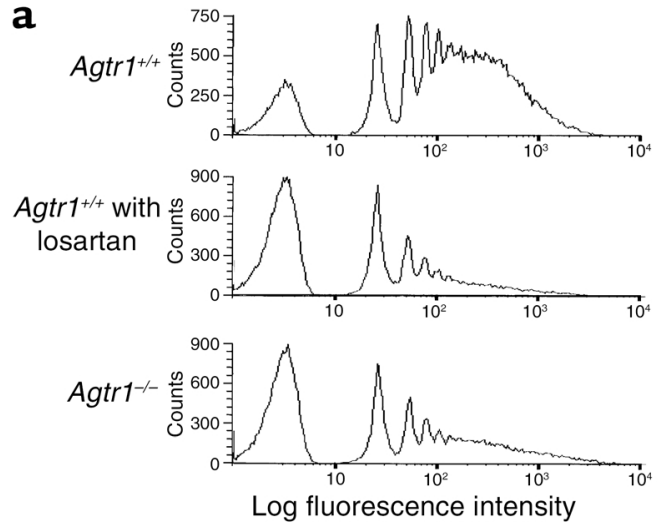

C

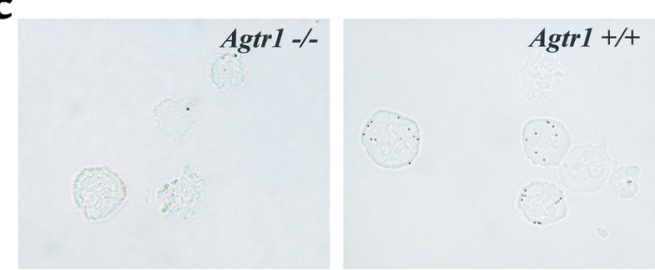

b

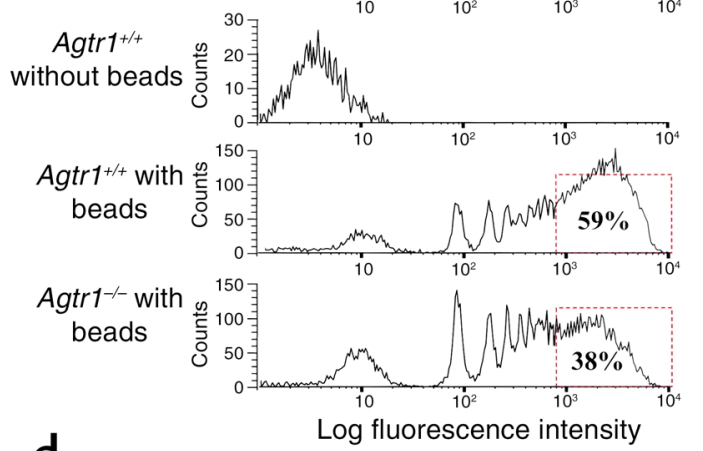

d

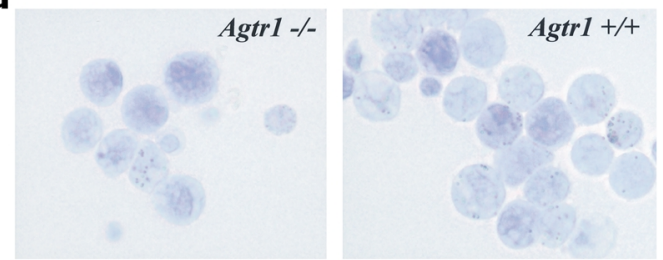

\section{Figure 8}

Phagocytic activity of Agtr1 $1^{-/-}$and Agtr1 ${ }^{+/+}$macrophages. (a) Fluorescent beads were injected into the peritoneal cavity after macrophage induction by thioglycollate, and the exudate was analyzed by flow cytometry for in vivo assay of phagocytosis. Histograms include Agtr $1^{+/+}$ macrophages, Agtr1 ${ }^{+/+}$macrophages treated with $10^{-6} \mathrm{M}$ losartan, and Agtr1 $1^{-/-}$macrophages. Three separate experiments were carried out, and representative results are shown. (b) Peritoneal macrophages were incubated in vitro with fluorescent beads, and the phagocytic activity was analyzed by flow cytometry. Histograms include fluorescent beads only, Agtr $1^{+/+}$macrophages incubated without fluorescent beads, and Agtr1 $1^{+/+}$and Agtr1 $1^{-/}$macrophages incubated with fluorescent beads. The mean fluorescence intensity level (lin Mean $\left.X\right)$ of each peak is shown for fluorescent beads only. The percentages of macrophages with multiple beads (more than eight) are shown next to the Agtr $1^{+/+}$ and Agtr1 ${ }^{-1-}$ histograms, respectively. R1 denotes the peak derived from one fluorescent bead; R2, two beads; R3, three beads; and R4, four beads. Three separate experiments were carried out, and representative results are shown. (c) In vitro phagocytosis of fluorescent beads by Agtr1 $1^{-/-}$(left) and Agtr1 $1^{++}$(right) macrophages viewed under a light microscope. Magnification, $\times 400$. (d) In vivo phagocytosis of fluorescent beads by Agtr1 $1^{-/-}$(left) and Agtr1 ${ }^{+/+}$(right) macrophages. Magnification, $\times 400$.

UUO induction. In later stages of UUO, interstitial fibrosis was more pronounced in mice with Agtr1 $^{-/}$ marrow, whereas macrophage infiltration was less than that in mice with Agtr1 $1^{++}$marrow (Figure 4a). This histologically appreciable augmentation of fibrosis was confirmed independently by a biochemical determination of collagen content (Figure $4 b$ ). The gene expression of the profibrotic and antifibrotic molecules followed a temporal pattern that indicates enhanced fibrogenesis in mice with Agtr1 $1^{-/-}$marrow. Thus, mRNA levels for $\alpha 1$ (I) collagen, $\alpha 1$ (III) collagen, and TGF- $\beta$ as well as for MMP- 2 were greater in mice with Agtr $1^{-/-}$marrow 14 days after the induction of UUO, indicating that in later stages of UUO, both profibrotic and antifibrotic activities of the resident renal cells were heightened in wild-type mice with Agtr1 $1^{-/-}$marrow in association with reduced macrophage infiltration.

We assessed the histological alterations that are known to be associated with the evolution of renal fibrosis. Peritubular capillary density, an architecture that is affected by macrophage-derived $\operatorname{FGF}(30,36)$, was evaluated by immunostaining for CD31 (Figure $3 \mathrm{~b}$ ), and no significant difference was found between wild-type mice reconstituted with $A g t r 1^{+/+}$or $A g t r 1^{-/-}$ marrow, suggesting that alteration in angiogenesis is not involved in the increased interstitial fibrosis in mice with Agtr $1^{-/-}$marrow. A recent report indicated that transdifferentiation of tubular epithelial cells to myofibroblasts occurs in a mouse UUO model and contributes to the progression of renal fibrosis (37). In this regard, we performed immunostaining of $\alpha$-SMA (Figure 3c) and found no difference between the two groups of mice, indicating that alterations in the epithelial-mesenchymal transition are not associated with the increased fibrosis. Likewise, T-cell infiltration was equally unremarkable in the two groups.

Since macrophages are the dominant infiltrating cells in UUO (6) and a decrease in their quantity was associated with enhanced fibrosis, we focused on the features of macrophages. Analyses of peripheral blood showed that wild-type mice reconstituted with Agtr1 $1^{-/-}$marrow had a reduced number of circulating monocytes (Table 1). We also found that macrophage progenitors in bone marrow were reduced in mice with Agtr $1^{-/-}$marrow (Figure 6). Migrating activity of macrophages examined using MCP-1 and angiotensin II as chemotactic factors showed that in response to MCP-1, both $\mathrm{Agtr}^{+/+}$ and $A g t r 1^{-/-}$macrophages exhibited a similar migrating 
activity (Figure 7a), suggesting that the migrating activity of macrophages was not different. Moreover, no chemotactic response was seen with angiotensin II at $10^{-6} \mathrm{M}$. These findings indicate that decreased levels of circulating monocytes and bone marrow macrophage progenitors are one of the factors underlying the reduced infiltration of Agtr $1^{-/-}$macrophages found in the obstructed kidneys of mice reconstituted with Agtr $1^{-/-}$marrow. In addition, both $A g t r 1^{+/+}$and Agtr1 $1^{-/-}$ macrophages showed similar oxidative burst on stimulation (Figure 7b), suggesting that together with reduced macrophage infiltration, macrophage-mediated oxidative injury was less significant in wild-type mice with $A g t r 1^{-/-}$macrophages.

Further analyses revealed impaired phagocytic capability of Agtr1 1-- macrophages both in vitro and in vivo (Figure 8). We also found that in vivo administration of losartan to $\mathrm{Agtr}^{+/+}$mice reduced phagocytic capability of $\mathrm{Agtr}^{+/+}$macrophages to a level similar to that of Agtr $1^{-/-}$macrophages (Figure 8), suggesting that angiotensin II acts on the Agtr 1 on macrophages in vivo and stimulates phagocytic capability. In keeping with this notion, macrophages harvested from $\mathrm{Agtr}^{+/+}$ mice did not respond to either angiotensin II or losartan in vitro (Figure 8 ). These in vivo and in vitro observations are reminiscent of the previous demonstration by others that in vivo losartan treatment of hypercholesterolemic patients results in suppression of oxidized LDL uptake and decreased expression of the scavenger receptor CD36 (41). Furthermore, a previous report (42) showed that in experimental immune-complex nephritis, changes in the phagocytic activity of glomerular macrophages are in large part parallel to those in the phagocytic activity of peritoneal macrophages elicited by complete Freund adjuvant. Thus, our findings suggest that impaired phagocytic activity of $\mathrm{Agtr}^{-/-}$macrophages infiltrating the renal interstitium relates to the increased renal fibrosis.

Destructive effects of macrophages on the scarring of the glomerulus and interstitium have been speculated on the basis of the observed positive correlation between the quantity of infiltrating macrophages and tissue damage (7). Of note, this apparent correlation does not necessarily establish that infiltrating macrophages serve only as profibrotic elements. In order to ascertain the function of macrophages in the evolution of fibrosis, it is of critical importance to vary the number of the infiltrating macrophages in the presence of an identical obstructive insult and analyze the pattern of correlation between infiltrating macrophages and the degree of fibrosis. In this regard, systemic blockade of Agtr1 using angiotensin II antagonists or ACE inhibitors does not allow selective inhibition of angiotensin II actions on macrophages. A recent study of UUO-induced renal fibrosis in Agtr1adeficient mice (43) also did not dissociate macrophage infiltration and renal fibrosis. Our finding that attenuated macrophage accumulation is associated with accelerated renal fibrosis, therefore, provides a unique insight into the function of macrophages. It is notable that a salutary function of macrophages has been described in the process of wound healing $(12-15,44)$. Thus, macrophages have been shown to promote provisional ECM deposition in early stages of wound healing $(12,13)$, whereas in later stages, macrophages are thought to play an essential role through phagocytic clearance of ECM fragments and apoptotic cells (13, 44). In the setting of renal fibrosis, Thomas et al. (44) showed that interstitial macrophages promote the clearance of apoptotic cells. Collectively, a possibility arises that diminution in the quantity and/or quality of functioning macrophages, as seen in the present study, leads to sustained profibrotic activity in the resident renal cells. In line with this notion, TGF- $\beta$ mRNA expression was found to increase in mice reconstituted with $\mathrm{Agtr}^{-/-}$marrow, whereas no further increase was observed in mice carrying Agtr $1^{+/+}$marrow.

In summary, we have shown that the Agtr1 on macrophages functions in vivo to attenuate fibrogenetic processes and to preserve the renal parenchymal architecture. Our data suggest that through Agtr1 angiotensin II affects the quantity and phagocytic activity of macrophages. This mechanism may be the first line of defense after various types of injury, allowing the healing process to take place. Should this defense mechanism be overwhelmed, the response of the resident cells will proceed down the path of progressive tissue injury. The methodology of bone marrow transplantation with genetically engineered stem cells used in this study has unmasked this new function of angiotensin II in the macrophage.

\section{Acknowledgments}

This work was supported by National Institutes of Health grants DK-44757 and DK-37868, the Baxter Healthcare Corporation (Extramural Grant Program), and the Research for the Future Program of the Japan Society for the Promotion of Science. A part of this study was presented in abstract form at the 33rd and 34th Annual Meeting of the American Society of Nephrology. We thank Cathy Zhi Qi Xu, Ellen Donnert, and Catherine Allen for their excellent technical assistance.

\footnotetext{
1. Anderson, S., Meyer, T.W., Rennke, H.G., and Brenner, B.M. 1985. Control of glomerular hypertension limits glomerular injury in rats with reduced renal mass. J. Clin. Invest. 76:612-619.

2. Lewis, E.J., Hunsicker, L.G., Bain, R.P., and Rohde, R.D. 1993. The effect of angiotensin-converting-enzyme inhibition on diabetic nephropathy. N. Engl.J. Med. 329:1456-1462.

3. Marre, M., et al. 1997. Contribution of genetic polymorphism in the renin-angiotensin system to the development of renal complications in insulin-dependent diabetes. Genetique de la Nephropathie Diabetique (GENEDIAB) study group. J. Clin. Invest. 99:1585-1595.

4. Holdsworth, S.R., Neale, T.J., and Wilson, C.B. 1981. Abrogation of macrophage-dependent injury in experimental glomerulonephritis in the rabbit. Use of an antimacrophage serum. J. Clin. Invest. 68:686-698.

5. Van Goor, H., van der Horst, M.L., Fidler, V., and Grond, J. 1992. Glomerular macrophage modulation affects mesangial expansion in the rat after renal ablation. Lab. Invest. 66:564-571.

6. Schreiner, G.F., Harris, K.P., Purkerson, M.L., and Klahr, S. 1988. Immunological aspects of acute ureteral obstruction: immune cell infiltrate in the kidney. Kidney Int. 34:487-493.
} 
7. Van Goor, H., et al. 1994. Macrophages and renal disease. Lab. Invest. 71:456-464

8. Schreiner, G.F. 1991. The role of the macrophage in glomerular injury. Semin. Nephrol. 11:268-275.

9. Klahr, S., Ishidoya, S., and Morrissey, J. 1995. Role of angiotensin II in the tubulointerstitial fibrosis of obstructive nephropathy. Am. J. Kidney Dis. 26:141-146

10. Ishidoya, S., Morrissey, J., McCracken, R., Reyes, A., and Klahr, S. 1995. Angiotensin II receptor antagonist ameliorates renal tubulointerstitial fibrosis caused by unilateral ureteral obstruction. Kidney Int. 47:1285-1294.

11. Diamond, J.R., and Pesek-Diamond, I. 1991. Sublethal X-irradiation during acute puromycin nephrosis prevents late renal injury: role of macrophages. Am. J. Physiol. 260:F779-F786.

12. Leibovich, S.J., and Ross, R. 1975. The role of the macrophage in wound repair: a study with hydrocortisone and antimacrophage serum. Am.J. Pathol. 78:71-100.

13. Singer, A.J., and Clark, R.A. 1999. Cutaneous wound healing. N. Engl.J. Med. 341:738-746.

14. Danon, D., Kowatch, M.A. and Roth, G.S. 1989. Promotion of wound repair in old mice by local injection of macrophages. Proc. Natl. Acad. Sci. USA. 86:2018-2020.

15. DiPietro, L.A., Burdick, M., Low, O.E., Kunkel, S.L., and Strieter, R.M. 1998. MIP-1a as a critical macrophage chemoattractant in murine wound repair. J. Clin. Invest. 101:1693-1698.

16. Foris, G., Dezso, B., Medgyesi, G.A., and Fust, G. 1983. Effect of angiotensin II on macrophage functions. Immunology. 48:529-535.

17. Okamura, A., et al. 1999. Upregulation of renin-angiotensin system during differentiation of monocytes to macrophages. J. Hypertens. 17:537-545.

18. Yanagitani, Y., et al. 1999. Angiotensin II type 1 receptor-mediated peroxide production in human macrophages. Hypertension. 33:335-339.

19. Lijnen, P., Fagard, R., and Petrov, V. 1997. Cytosolic calcium changes induced by angiotensin II in human peripheral blood mononuclear cells are mediated via angiotensin II subtype 1 receptors. J. Hypertens. 15:871-876

20. Nataraj, C., et al. 1999. Angiotensin II regulates cellular immune responses through a calcineurin-dependent pathway. J. Clin. Invest. 104:1693-1701.

21. Rodgers, K.E., Xiong, S., Steer, R., and diZerega, G.S. 2000. Effect of angiotensin II on hematopoietic progenitor cell proliferation. Stem Cells. 18:287-294

22. Linton, M.F., Atkinson, J.B., and Fazio, S. 1995. Prevention of atherosclerosis in apolipoprotein E-deficient mice by bone marrow transplantation. Science. 267:1034-1037.

23. Yoshida, H., et al. 2001. Isoform-specific effects of apolipoprotein E on atherogenesis: gene transduction studies in mice. Circulation Res. 104:2820-2825.

24. Matsusaka, T., et al. 1996. Chimeric mice carrying "regional" targeted deletion of the angiotensin type $1 \mathrm{~A}$ receptor gene. Evidence against the role for local angiotensin in the in vivo feedback regulation of renin synthesis in juxtaglomerular cells. J. Clin. Invest. 98:1867-1877.

25. Yoshida, H., et al. 1992. Analysis of the evolution of angiotensin II type 1 receptor gene in mammals (mouse, rat, bovine and human). Biochem. Biophys. Res. Commun. 186:1042-1049.
26. Chen, X., et al. 1997. Targeting deletion of angiotensin type $1 \mathrm{~B}$ receptor gene in the mouse. Am. J. Physiol. 272:F299-F304.

27. Ma, J., et al. 1998. Accelerated fibrosis and collagen deposition develop in the renal interstitium of angiotensin type 2 receptor null mutant mice during ureteral obstruction. Kidney Int. 53:937-944.

28. Miyazaki, Y., Tsuchida, S., Fogo, A., and Ichikawa, I. 1999. The renal lesions that develop in neonatal mice during angiotensin inhibition mimic obstructive nephropathy. Kidney Int. 55:1683-1695.

29. DeLisser, H.M., Newman, P.J., and Albelda, S.M. 1994. Molecular and functional aspects of PECAM-1/CD31. Immunol. Today. 15:490-495.

30. Kang, D.H., et al. 2001. Impaired angiogenesis in the remnant kidney model: I. Potential role of vascular endothelial growth factor and thrombospondin-1. J. Am. Soc. Nephrol. 12:1434-1447.

31. Ma, L.J., et al. 2000. Regression of sclerosis in aging by an angiotensin inhibition-induced decrease in PAI-1. Kidney Int. 58:2425-2436.

32. Lieschke, G.L., et al. 1994. Mice lacking granulocyte colony-stimulating factor have chronic neutropenia, granulocyote and macrophage progenitor cell deficiency, and impaired neutrophil mobilization. Blood. 84:1737-1746.

33. Scott, R.S., et al. 2001. Phagocytosis and clearance of apoptotic cells is mediated by MER. Nature. 411:207-211.

34. Djaldetti, M., et al. 1997. A simple method for evaluation of latex phagocytosis by rat peritoneal macrophages. Acta Haematol. 98:56-57.

35. Yokota, T., et al. 2000. Adiponectin, a new member of the family of soluble defense collagens, negatively regulates the growth of myelomonocytic progenitors and the functions of macrophages. Blood. 96:1723-1732.

36. Ohashi, R., Shimizu, A., Masuda, Y., Ishizuka, M., and Yamanaka, N. 2000. Angiogenesis and subsequent regression of peritubular capillary (PTC) in the unilateral ureteral obstruction (UUO) model. J. Am. Soc. Nephrol. 11:514A. (Abstr.)

37. Yang, J., and Liu, Y. 2001. Dissection of key events in tubular epithelial to myofibroblast transition and its implications in renal interstitial fibrosis. Am. J. Pathol. 159:1465-1475.

38. Diamond, J.R., Kees-Folts, D., Ding, G., Frye, J.E., and Restrepo, N.C. 1994. Macrophages, monocyte chemoattractant peptide- 1 , and TGF- $\beta 1$ in experimental hydronephrosis. Am.J. Physiol. 266:F926-F933.

39. Kintsher, U., et al. 2001. Angiotensin II induces migration and Pyk2/paxillin phosphorylation of human monocytes. Hypertension. 37:587-593.

40. Ricardo, S.D., and Diamond, J.R. 1998 The role of macrophages and reactive oxygen species in experimental hydronephrosis. Sem. Nephrol. 18:612-621.

41. Hayek, T., Aviram, M., Heinrich, R., Sakhnini, E., and Keidar, S. 2000. Losartan inhibits cellular uptake of oxidized LDL by monocytemacrophages from hypercholesterolemic patients. Biochem. Biophys. Res. Commun. 273:417-420.

42. Moxey-Mims, M.M., and Noble, B. 1994. Glomerular macrophage phagocytic activity in experimental immune complex nephritis. Kidney Int. 45:1326-1332.

43. Satoh, M., et al. 2001. Renal interstitial fibrosis is reduced in angiotensin II type 1a receptor-deficient mice. J. Am. Soc. Nephrol. 12:317-325.

44. Thomas, S.E., et al. 1998. Accelerated apoptosis characterizes cyclosporine-associated interstitial fibrosis. Kidney Int. 53:897-908. 